\title{
Effects of low-intensity ultrasound combined with low-dose carboplatin in an orthotopic hamster model of tongue cancer: A preclinical study
}

\author{
HAI-XIA LI ${ }^{1 *}$, JIN-HUA ZHENG ${ }^{2 *}$, LIANG JI ${ }^{2}$, GUAN-YAO LIU ${ }^{2}$, YV-KUN LV ${ }^{2}$, DAN YANG ${ }^{1}$, \\ ZHENG HU $^{3}$, $\mathrm{HE} \mathrm{CHEN}^{1}$, FENG-MIN ZHANG ${ }^{4}$ and WENWU CAO ${ }^{3,5}$ \\ Departments of ${ }^{1}$ Forensic Medicine and ${ }^{2}$ Anatomy, Harbin Medical University, Harbin, Heilongjiang 150081; \\ ${ }^{3}$ Laboratory of Sono- and Phototheranostic Technologies, Harbin Institute of Technology, Harbin, Heilongjiang 150080; \\ ${ }^{4}$ Department of Microbiology, Harbin Medical University, Harbin, Heilongjiang 150081, P.R. China; \\ ${ }^{5}$ Materials Research Institute and Department of Mathematics, Pennsylvania State University, University Park, PA 16802, USA
}

Received June 20, 2017; Accepted February 6, 2018

DOI: $10.3892 / o r .2018 .6262$

\begin{abstract}
Low-intensity ultrasound (LIUS) combined with chemotherapy is an innovative modality for cancer treatment, but its effect on orthotopic carcinoma remains unknown. Our previous study revealed that LIUS enhanced the growth inhibitory effects of several chemotherapeutic drugs in nude mice with transplanted tumors. In the present study, we used 7,12-dimethylbenz(alpha)anthracene to induce orthotopic tongue carcinogenesis in hamsters. We used the first-line chemotherapy drug for tongue cancer, carboplatin (CBP) in combination with LIUS to investigate the synergistic effect. The results revealed that LIUS combined with low-dose CBP enhanced the inhibitory effects of CBP on tumor growth, prolonged survival, and did not increase the incidence of sideeffects. It also enhanced the inherent DNA damage caused by $\mathrm{CBP}$, suppressed the expression of the DNA repair proteins $O^{6}$-methylguanine DNA methyltransferase (MGMT) and Chk1, and increased the expression of DNA damage-inducible protein GADD $45 \alpha$. Furthermore, compared with CBP alone, LIUS combined with CBP reduced the expression of cyclin D1 and cyclin B1, induced the expression of caspase-3, cleaved
\end{abstract}

Correspondence to: Professor Wenwu Cao, Materials Research Institute and Department of Mathematics, Pennsylvania State University, N-317 Millennium Science Complex, Pollock Road, University Park, PA 16802, USA

E-mail:dzk@psu.edu

Professor Feng-Min Zhang, Department of Microbiology, Harbin Medical University, 157 Baojian Road, Harbin, Heilongjiang 150081, P.R. China

E-mail: fengminzhang@ems.hrbmu.edu.cn

${ }^{*}$ Contributed equally

Key words: low-intensity ultrasound, carboplatin, orthotopic tongue cancer, MGMT, Chk1, GADD45 $\alpha$ caspase-3, caspase-8, Bax, and Bak, and inhibited the expression of Bcl-2. Examination of clinical samples revealed that MGMT, Chk1, and Gadd45 $\alpha$ were higher in OTSCC than in adjacent normal tissue. Hence, our results indicated that LIUS enhanced the ability of low-dose CBP to damage DNA in an orthotopic hamster model of tongue cancer, induced apoptosis, inhibited tumor growth and progression, while it did not increase the toxic side-effects of the drug, suggesting additional clinical benefits for patients treated with the combination of CBP with LIUS.

\section{Introduction}

Large areas of surgical resection or high doses of chemotherapy to treat oral squamous cell carcinoma (OSCC) lead to serious clinical complications and poor prognosis $(1,2)$. Thus, to improve the quality of life of OSCC patients, better clinical treatment strategies with fewer lesions and complications are warranted.

Ultrasound (US) is already known to be a chemosensitizer (3-10). US has immediate sonoporation effects and can achieve the same clinical effect when a lower dose of the same drug is used (11), resulting in reduced side-effects. Low-intensity ultrasound (LIUS) can increase the permeability of the plasma membrane and improve the effects of anticancer drugs without causing damage to the entire cell, both in vivo and in vitro (3). LIUS-mediated chemosensitivity is mainly achieved by increasing the accumulation of intracellular drugs, although other mechanisms may also be involved $(4,9)$. LIUS also plays a role in targeted chemotherapy, the direct release of anticancer drugs, and the induction of apoptosis and necrosis of cells $(12,13)$.

We previously reported that LIUS $\left(1.1 \mathrm{MHz}, 1.0 \mathrm{~W} / \mathrm{cm}^{2}\right.$, $10 \%$ duty cycle) combined with low doses of scutellarin or 5-fluorouracil produced enhanced synergistic antitumor effects in vitro and in vivo, prolonged the survival time of mice without significant cytotoxicity in normal cells $(3,4)$. LIUS can limit the side-effects to the pathological site and minimize damage to surrounding normal tissues. This treatment modality has 
great potential in clinical applications. However, to date, LIUS combined with chemotherapeutic agents has not yet been used clinically, possibly because the reported efficacy depends on the drug and tumor model and thus, further investigation is warranted (6).

Carboplatin (CBP) as the first-line chemotherapeutic agent for OSCC, is a second-generation platinum drug. Small inorganic platinum compounds move across cancer cell membranes and bind to the DNA, forming a variety of inter-strand and intra-strand cross-links between the platinum compounds and nucleotides, which trigger a series of intracellular events that ultimately lead to the cell death (14-16). However, the clinical utility of these drugs has been limited by their harmful side-effects, which include nephrotoxicity, myelosuppression, nausea, and vomiting (14). In addition, the efficacy of platinum-based drugs is often compromised by the inherent or acquired resistance of cancer cells (17). The mechanisms underlying platinum resistance are very complex, and include decreased drug uptake, increased drug efflux, activation of detoxifying systems, activation of DNA repair mechanisms, and the evasion of drug-induced apoptosis $(15,17)$. Studies have revealed that the reduced accumulation of intracellular platinum is a prominent feature of platinum resistance (15). Whether US can enhance the cytotoxicity of platinum-based drugs remains a subject of debate. Saad and Hahn (18) reported that US did not enhance the effects of CDDP in Chinese hamster ovary cells, however Yu et al (9) demonstrated that US increased CDDP-induced DNA damage. Therefore, further studies are warranted to clarify whether this combined treatment modality can serve as a non-invasive method to improve the local permeability and targeting of platinum-based drugs in order to increase the clinical treatment efficacy.

To the best of our knowledge, the development of preclinical drugs and therapeutic modalities mostly rely upon the subcutaneous inoculation of tumor cells in immunodeficient mice to establish a tumor xenograft model (19). However, subcutaneous models do not sufficiently represent the clinical features of tumors, particularly with respect to metastasis development and response to treatment $(20,21)$. Chemically-induced orthotopic models are commonly used for the preclinical and translational studies of compounds and therapies because they mimic many aspects of human diseases, provide reproducible results and allow evaluation of the systemic effects of treatments. Our research team previously demonstrated that hamster tongue mucosa underwent gradual changes from hyperplasia, carcinoma in situ to early invasive carcinoma, when exposed to 7,12-dimethylbenz(alpha)anthracene (DMBA) $(22,23)$. The pathogenesis of this tongue cancer model is similar to human tongue carcinoma.

In the present study, DMBA was used to induce an orthotopic model of tongue cancer, and we used very low acoustic intensity that complied with current clinical safety regulations and a current clinical chemotherapeutic drug CBP $(14,17)$ to investigate whether LIUS combined with low-dose CBP enhances the anticancer efficacy and decreases the side-effects of CBP.

\section{Materials and methods}

Tumor model and chemicals. All animal experimental procedures were approved by the Laboratory Animal
Committee of Harbin Medical University (Harbin, China). Four-week-old male hamsters (35-55 g) from the Beijing Vital River Laboratory Animal Technology Co., Ltd. (Beijing, China) were housed under specific pathogen-free conditions. To create an orthotopic tongue cancer model, a 1.5\% DMBA acetone solution (Sigma-Aldrich, St. Louis, MO, USA) was applied to the left margin of the anterior tongue in a scratched area, three times a week, and hamsters were fasted for $2 \mathrm{~h}$ after applying DMBA to avoid damage produced by DMBA to the other alimentary structures other than the tongue according to a previously published method by Fujita et al (24), until a tumor was formed in the tongue ( $~ 8$ weeks). Eight weeks after DMBA application (tumor diameter, 3 4 mm), the tongue cancer grew rapidly $(23,25)$ and treatment began. The carboplatin injection was purchased from Qilu Pharmaceutical Co., Ltd. (Jinan, China) and diluted with $0.9 \%$ sodium chloride solution to the appropriate concentration for injection. Rabbit polyclonal anti-Chk1 (cat. no. WL01674; IHC, 1:100; WB, 1:500), rabbit polyclonal anti-proliferating cell nuclear antigen (PCNA; cat. no. WL0341; IHC, 1:50), rabbit polyclonal anti-Bcl-2 (cat. no. WL01556; IHC, 1:100; WB, 1:500), rabbit polyclonal anti-cyclin B1 (cat. no. WL01760; IHC, 1:100; WB, 1:500), rabbit polyclonal anti-p53 (cat. no. WL01919; WB, 1:500), rabbit polyclonal anti-cleaved caspase-3 (cat. no. WL01992; WB, 1:500), rabbit polyclonal anti-phospho-p53 (cat. no. WL02504; WB, 1:500), rabbit polyclonal anti-caspase-8 (cat. no. WL00659; IHC, 1:100; WB, 1:500), rabbit polyclonal anti-Bax (cat. no. WL01637; IHC, 1:100; WB, 1:500), rabbit polyclonal anti-p21 (cat. no. WL0362; WB, 1:500), and rabbit polyclonal anti- $\beta$ actin (cat. no. WL01845; WB, 1:1,000) were purchased from Wanleibio Co., Ltd. (Changchun, China). Rabbit polyclonal anti-Bak (cat. no. PB0506; IHC, 1:100; WB, 1:500), rabbit polyclonal anti-caspase-3 (cat. no. BA3968; IHC, 1:100; WB, 1:300) and rabbit polyclonal anti-cyclin D1 (cat. no. BA0770; IHC, 1:100; WB, 1:300) were purchased from Wuhan Boster Bioengineering Co., Ltd. (Wuhan, China), rabbit polyclonal anti-MGMT (cat. no. BS1002R; IHC, 1:500; WB, 1:1,000) was purchased from Beijing Biosynthesis Biotechnology Co., Ltd. (Beijing, China), Mouse monoclonal anti- $\gamma-\mathrm{H} 2 \mathrm{AX}$ (cat. no. ab26350; IHC, 1:100; WB, 1:1,000) was provided by Abcam (Cambridge, UK) and rabbit polyclonal anti-Gadd $45 \alpha$ (cat. no. E2A6622; IHC, 1:100; WB, 1:300) was purchased from EnoGene (Nanjing, China).

US device and treatment protocol. Fig. 1A displays the US treatment system developed by the Condensed Matter Science and Technology Institute, Harbin Institute of Technology (Harbin, China). In the animal treatment experiments, a toneburst US signal generated by a $5.0 \mathrm{~cm}$ diameter piezoelectric transducer with a center frequency of $1.0 \mathrm{MHz}$ was applied through a tapered aluminum buffer head whose front surface ( $5 \mathrm{~mm}$, diameter) was put directly in contact with the tongue cancer site using an ultrasonic couplant. US intensity was measured in degassed water using an HNC-1000 needle-type hydrophone $(0.1 \mathrm{~cm}$ active element size, 1 20 MHz bandwidth) (Onda Corporation, Sunnyvale, CA, USA). The sound pressure level distribution pattern was calculated by finite element simulations using COMSOL software (26). The US frequency was $1.0 \mathrm{MHz}$, provided in tone burst mode with a 
duty cycle of $20 \%$ and a repetition frequency of $100 \mathrm{~Hz}$; the ultrasonic intensity level was $0.89 \mathrm{~W} / \mathrm{cm}^{2}$. We chose $20 \mathrm{mg} / \mathrm{kg}$ as a low dose for the study (Fig. 1B), which can slow the growth of the hamster tongue cancer but cannot stop the tumor growth. The hamsters were randomly divided into five groups: Untreated control (control group, $\mathrm{n}=10$ ); LIUS treatment (U group, $0.89 \mathrm{~W} / \mathrm{cm}^{2}$ intensity, $1.0 \mathrm{MHz}$ frequency, $20 \%$ duty factor, 15 min duration; $\mathrm{n}=10$ ); intraperitoneal injection of $20 \mathrm{mg} / \mathrm{kg}$ CBP (CB20 group, $\mathrm{n}=10$ ); intraperitoneal injection of $40 \mathrm{mg} / \mathrm{kg}$ CBP (CB40 group, $\mathrm{n}=10)$; and LIUS combined with intraperitoneal injection of CBP (U+CB group, $20 \mathrm{mg} / \mathrm{kg}$ CBP administration $1 \mathrm{~h}$ before LIUS treatment; $\mathrm{n}=10$ ). All animals were treated twice a week for a total of 8 weeks and then the therapy was terminated. The tumor volumes and body weights were assessed twice a week. The tumor volume was calculated using the formula: $\mathrm{V}=(\pi / 6) \times \mathrm{L} \times \mathrm{S}^{2}$, where $\mathrm{L}$ and $\mathrm{S}$ are the long and short diameters, respectively. At the end of the treatment, five hamster tongue cancer tissues were excised and abdominal aorta blood was drawn from each group for analysis; the remaining five hamsters were observed for survival until day 90, and then all hamsters were sacrificed.

Clinical samples. Archived paraffin-embedded oral tongue squamous cell carcinoma tissues (OTSCC) and matched adjacent normal tissues were obtained from 48 patients who had undergone surgical excision at Harbin Medical University Stomatological Hospital between January 2000 and December 2005 (27). All of the patients provided informed consent, and the study was approved by the Research Ethics Committee of Harbin Medical University. All patients underwent potentially curative surgery without preoperative therapy. Patient clinical characteristics were previously described (27).

Immunohistochemistry. Tumors were excised, fixed in $4 \%$ paraformaldehyde, dehydrated with a graded ethanol series, cleared in dimethylbenzene, and embedded in paraffin. Next, tissue blocks were cut into $4-\mu \mathrm{m}$ sections and mounted on glass slides, and then routinely dewaxed and rehydrated. Antigens were retrieved in $10 \mathrm{mM}$ citrate buffer $(\mathrm{pH}$ 6.0) for $15 \mathrm{~min}$ in a pressure cooker. Tissue sections were treated with endogenous peroxidase at room temperature. After blocking in $1 \%$ bovine serum albumin for $30 \mathrm{~min}$, the sections were stained with appropriate primary antibodies and incubated overnight at $4^{\circ} \mathrm{C}$. Subsequently, the sections were incubated with corresponding secondary antibodies (immediate-use goat antirabbit IgG horseradish-peroxidase polymers; cat. no. PV-6001; Zhongshan Golden Bridge Biotechnology Co., Ltd., Beijing, China) for $30 \mathrm{~min}$ at $37^{\circ} \mathrm{C}$. The antibody reaction was visualized using diaminobenzidine chromogen (cat. no. ZLI-9018; Zhongshan Golden Bridge Biotechnology). Finally, all the slides were counterstained with hematoxylin. For protein expression analysis, 10 areas were randomly selected under a microscope at a magnification of x200. Image Pro Plus 6.0 (Media Cybernetics, Inc., Bethesda, MD, USA) was used to quantify the intensity and extent of immunopositive expression in cells with integrated optical density (IOD) values. IOD values were expressed as the mean \pm SD per tissue examined.

Western blotting. The tissue was cut into small pieces on ice, lysed in cell lysis buffer (cat. no. P0013; Beyotime Institute of
Biotechnology, Nantong, China) in the presence of the Complete Mini Proteinase Inhibitor Cocktail (Roche Diagnostics, Indianapolis, IN, USA), resolved on $10 \%$ SDS-PAGE gels, and electrotransferred to nitrocellulose membranes. After blocking in Tris-buffered saline and Tween-20 (TBS-T) containing 5\% non-fat dry milk, the membranes were incubated overnight at $4^{\circ} \mathrm{C}$ with primary antibodies against the target proteins. After washing twice with TBS-T, the membranes were incubated with horseradish peroxidase-conjugated secondary antibodies for $1 \mathrm{~h}$. The protein levels were detected using a fully automated chemiluminescence imaging analysis system (Tanon Science and Technology Co., Ltd., Beijing, China).

Statistical analysis. Three different observers assessed the primary tumor volume, and two were blind to the research groups. The results were expressed as the mean \pm standard error of the mean (SEM). Statistical differences were evaluated using one-way analysis of variance (ANOVA) followed by Dunnett's test. Differences between any two groups were assessed by the Student Newman-Keuls test, with P-values $<0.05$ considered as statistically significant. Statistical evaluation was performed using SPSS 22.0 (IBM Corp., Armonk, NY, USA).

\section{Results}

LIUS enhances the anticancer effects of CBP and prolonged survival. Following corresponding treatment of hamsters in different groups, tumor images of each group were captured (Fig. 2A). The results revealed that the tumor volume in the $\mathrm{U}+\mathrm{CB}$ group was the smallest among the 5 groups, and the tumor volume changes were 40, 42, 15, 3 and 6-fold for the control, U, CB20, U+CB, and CB40 groups, respectively (Fig. 2B) at the end of treatment compared with the tumor volume at the time of treatment initiation. The results revealed that the growth rate of the orthotopic tongue carcinoma in the $\mathrm{U}+\mathrm{CB}$ group was only $1 / 5$ th of that of the group treated with $20 \mathrm{mg} / \mathrm{kg}$ CBP alone (CB20 group; $\mathrm{P}<0.05$ ).

To ascertain the cell proliferation status of tumor cells, we examined the expression of PCNA in the tongue carcinoma tissues under different treatments. The expression level of PCNA was high in the LIUS-treated tissue, and there was no significant difference between the LIUS-treated and the control groups. With the increase of the CBP dosage, the PCNA expression level gradually decreased and was further inhibited after combination with LIUS compared with CBP treatment alone (Fig. 2C). These results indicated that LIUS can enhance the inhibitory effects of CBP on the proliferation of hamster orthotopic tongue cancer cells. In our previous study, we demonstrated that LIUS increased the accumulation of drugs in cancer cells (4); therefore, we speculated that LIUS may increase accumulation of intracellular CBP in hamster orthotopic tongue cancer in this study. There were no significant differences in body weight between the drugtreated groups (20 and $40 \mathrm{mg} / \mathrm{kg} \mathrm{CBP}$ ) and the control group; all ultrasonic treatments (U, U+CB) led to slight but nonsignificant decreases in body weight compared to the control group (Fig. 2D; $\mathrm{P}=0.998$ ). Side-effects were not observed in any group. Since the ultrasonic treatment needs to be performed under anesthesia, we hypothesized that the decrease in body weight was related to the anesthesia. No significant weight loss 



Figure 1. (A) Schematic diagram of the ultrasound treatment system. The ultrasound signal was applied through a tapered aluminium head with its front surface placed directly in contact with the tumor site using ultrasonic couplant. (B) Tumor sites of the hamster's tongue were treated with CBP at a dosage of 10, 20 and $40 \mathrm{mg} / \mathrm{kg}$, respectively. Data were represented as the mean $\pm \mathrm{SD}(\mathrm{n}=6) . \mathrm{CPB}$, carboplatin.
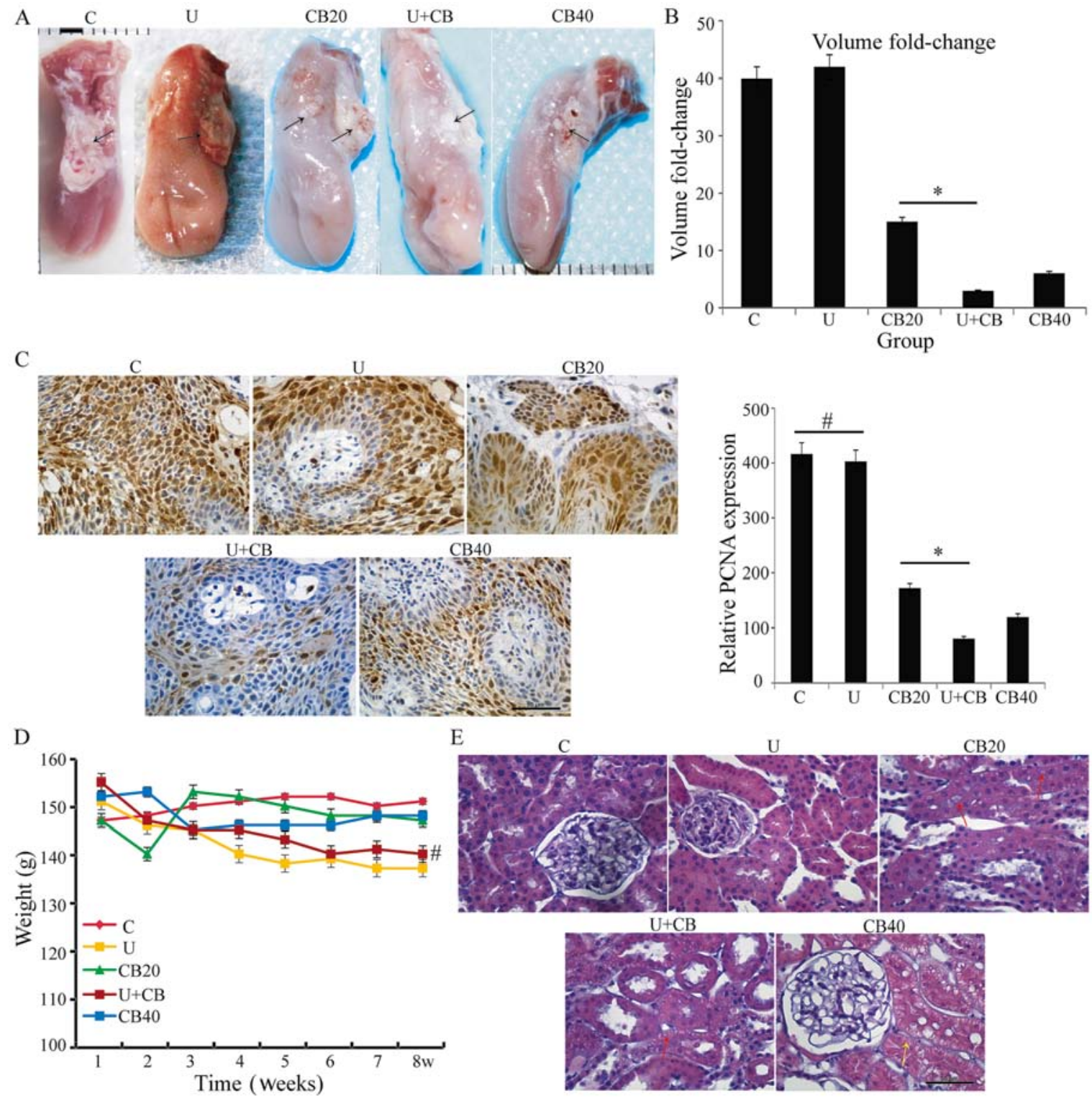

Figure 2. LIUS enhanced the anticancer effects of CBP in hamsters with orthotopic tongue carcinoma. (A) Gross view of a representative orthotopic tongue cancer. The tumor mass (arrowhead) in the C, U, and CB20 groups was more evident than that in the U+CB group; Scale bar, $2 \mathrm{~mm}$. (B) Tumor volume changes in the orthotopic hamster model of tongue cancer (data are expressed as the mean $\pm \mathrm{SD} ; \mathrm{n}=5$ hamsters/group); $\mathrm{CB} 20$ vs. $\mathrm{U}+\mathrm{CB}$, "P $<0.05$. (C) $\mathrm{PCNA}$ expression in the C, U, CB20, CB40, and U+CB groups. Data are expressed as the mean \pm SD. Statistical significance was determined by one-way ANOVA followed by Dunnett's test. "P<0.05; " no significance between groups. Scale bar, $50 \mu \mathrm{m}$. (D) Body weight curve in the C, U, CB20, CB40, and U+CB groups. "No significance between U+CB vs. C. (E) Representative pathological results of the kidney tissue; renal tubular epithelial cells exhibiting slight edema (red arrows) and severe edema (yellow arrow) were observed in the CB40 group. C, control group; U, low-level ultrasound treatment group; CB20, $20 \mathrm{mg} / \mathrm{kg}$ CBP treatment group; CB40, $40 \mathrm{mg} / \mathrm{kg}$ CBP treatment group; U+CB, low-level ultrasound combined with the $20 \mathrm{mg} / \mathrm{kg}$ CBP treatment group. 
Table I. Systemic toxicity determined by blood tests.

\begin{tabular}{|c|c|c|c|c|c|c|}
\hline Group & $\begin{array}{c}\text { ER } \\
\left(\mathrm{x} 10^{12} / 1\right)\end{array}$ & $\begin{array}{c}\mathrm{LE} \\
\left(\mathrm{x} 10^{9} / 1\right)\end{array}$ & $\begin{array}{c}\mathrm{PL} \\
\left(\mathrm{x} 10^{9} / 1\right)\end{array}$ & $\begin{array}{c}\mathrm{CR} \\
(\mathrm{mg} / \mathrm{dl})\end{array}$ & $\begin{array}{c}\text { UN } \\
(\mathrm{mg} / \mathrm{dl})\end{array}$ & $\begin{array}{c}\mathrm{UA} \\
(\mu \mathrm{mol} / \mathrm{l})\end{array}$ \\
\hline Control & $3.2 \pm 0.2$ & $6.6 \pm 0.2$ & $698.4 \pm 8.1$ & $2.2 \pm 0.8$ & $25.9 \pm 3.8$ & $36.4 \pm 5.5$ \\
\hline US & $3.3 \pm 0.1$ & $6.3 \pm 0.8$ & $701.2 \pm 10.4$ & $2.0 \pm 0.7$ & $25.4 \pm 1.6$ & $42 \pm 4.6$ \\
\hline CBP20 & $3.6 \pm 0.1$ & $6.0 \pm 0.1$ & $675.2 \pm 5.0$ & $2.8 \pm 0.8$ & $83.0 \pm 5.0$ & $43.2 \pm 3.0$ \\
\hline CBP40 & $3.3 \pm 0.1$ & $5.8 \pm 0.1$ & $629.6 \pm 45.1$ & $4.4 \pm 1.1$ & $117.9 \pm 6.3$ & $50.8 \pm 4.3$ \\
\hline $\mathrm{CBP}+\mathrm{US}$ & $3.6 \pm 0.2$ & $6.3 \pm 0.1$ & $670.4 \pm 17.3$ & $3.0 \pm 0.7$ & $88.4 \pm 1.8$ & $45.4 \pm 3.5$ \\
\hline
\end{tabular}

Data are presented as the mean \pm SEM of five animals. Multiple comparisons were performed using one-way ANOVA followed by the Bonferroni test applied as the post hoc test for selected pairs. ER, erythrocyte; LE, leukocyte; PL, platelet; CR, creatinine; UN, urea nitrogen; UA, uric acid.

occurred in the CB40 group, indicating that this drug dose did not have significant effects on hamster growth.

To further examine possible side-effects of this drug, we drew blood from the abdominal aorta of the animals for blood and renal function tests. There was no significant differences in erythrocyte (ER), leukocyte (LE), and platelet (PL) counts between the $\mathrm{CB} 20$ and $\mathrm{U}+\mathrm{CB}$ groups (ER: $\mathrm{P}=0.999$; LE: $\mathrm{P}=0.014$; PL: $\mathrm{P}=0.991$; Table I). Although CBP treatment increased serum levels of creatinine (CR), urea nitrogen (UN), and uric acid (UA) levels compared with the control group, $\mathrm{U}+\mathrm{CB}$ treatment did not produce significant differences compared to the $\mathrm{CB} 20$ group (CR: $\mathrm{P}=0.988$; $\mathrm{UN}$ : $\mathrm{P}=0.149$; UA: $\mathrm{P}=0.831$; Table I). These findings revealed that LIUSenhanced the anticancer effects of CBP in vivo, and did not worsen the systemic toxicity of the drug.

The pathology of the kidney after different treatments is displayed in Fig. 2E. LIUS exposure did not cause any histopathological changes in the kidneys. There was slight edema of renal tubular epithelial cells in the CB20 and U+CB groups, and edema of renal tubular epithelial cells was observed in the CB40 group. Histopathological changes in the kidney resulting from the $\mathrm{U}+\mathrm{CB}$ treatment were similar to those observed in the group treated with $20 \mathrm{mg} / \mathrm{kg}$ CBP alone.

When the tumor grew to $\sim 4 \mathrm{~mm}$ in diameter volume, treatment was initiated and animal survival was monitored daily until day 90. The results were presented in a Kaplan-Meier graph (Fig. 3), and the median survival times for the five groups were $60,50,80,90$, and $>90$ days, respectively. The survival time was prolonged in the CB20, CB40, and U+CB groups compared with the control group $(\mathrm{P}<0.05)$. These data clearly indicated that LIUS combined with CBP was more effective than the treatment with $40 \mathrm{mg} / \mathrm{kg}$ CBP alone in prolonging the animal survival time $(\mathrm{P}=0.0159)$, extending the long-term survival rate presumably due to the more effective CBP delivery through LIUS-mediated tumor cell targeting. In addition, some hamsters were in a state of poor health and died during treatment. In future experiments, systematic investigation will be performed to determine whether hamsters died of complications or tumor metastasis.

LIUS treatment alone had no significant effects on the growth and proliferation of orthotopic tongue cancer cells. LIUS treatment alone did not significantly reduce the growth

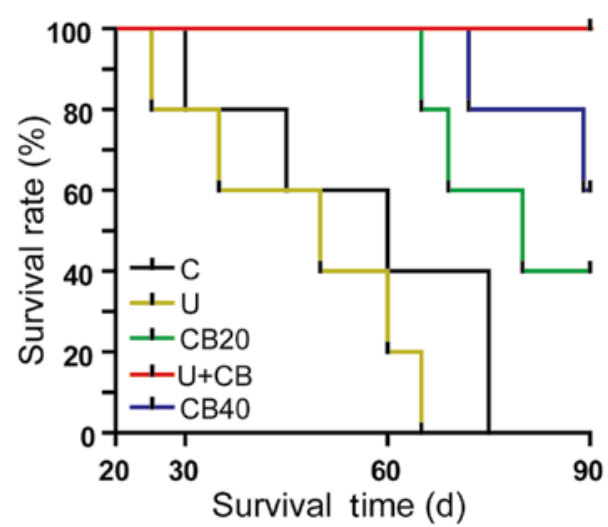

Figure 3. Survival curves in the hamster orthotopic tongue cancer model Survival time was prolonged in the group treated with $20 \mathrm{mg} / \mathrm{kg}$ CBP (CB20 group), $40 \mathrm{mg} / \mathrm{kg} \mathrm{CBP}$ (CB40 group), or a combination of LIUS and CBP $(\mathrm{U}+\mathrm{CB})$ compared with the control group, with the longest survival time observed in the U+CB group. $\mathrm{CPB}$, carboplatin; $\mathrm{C}$, control group; U, low-level ultrasound treatment group; CB20, $20 \mathrm{mg} / \mathrm{kg}$ CBP treatment group; CB40, $40 \mathrm{mg} / \mathrm{kg}$ CBP treatment group; U+CB, low-level ultrasound combined with the $20 \mathrm{mg} / \mathrm{kg}$ CBP treatment group.

in the xenograft model of human-tongue squamous carcinoma and hepatocellular carcinoma $(3,4)$. Carboplatin is not a sensitizer, and the ultrasound-mediated cavitation effect did not participate in the synergistic effect of $\mathrm{U}+\mathrm{CB}$. Therefore, in subsequent experiments, we did not evaluate tongue cancer tissue in the LIUS treatment group.

LIUS combined with CBP induces DNA damage. The antitumor activity of platinum-based chemotherapy is largely dependent on the DNA repair capacity of cancer cells, therefore, we examined the expression of DNA damage repair-related proteins in different treatment groups. First, the $\mathrm{U}+\mathrm{CB}$ therapy-induced DNA damage was investigated using IHC to detect $\gamma-\mathrm{H} 2 \mathrm{AX}$. As shown in Fig. 4A, $\gamma$-H2AX expression was revealed in the nucleus of the CBP-treated group, and a higher $\gamma-\mathrm{H} 2 \mathrm{AX}$ expression level was revealed in the $\mathrm{U}+\mathrm{CB}$ group compared to the CBP alone group. MGMT was highly expressed in the control group. Its expression decreased with the increase of CBP concentration and was the lowest in the U+CB group (Fig. 4). We observed the expression of Gadd $45 \alpha$ 



Figure 4. LIUS and CBP induce DNA damage and cell cycle arrest. (A) $\gamma$-H2AX, MGMT, Gadd45 $\alpha$, Chk1, cyclin D1, and cyclin B1 expression was evaluated by immunohistochemical staining. Representative data from three independent experiments are shown. Scale bar, $50 \mu \mathrm{m}$. (B) The expression levels of MGMT, Gadd45 $\alpha$, Chk1, cyclin D1, cyclin B1, caspase-3, and cleaved caspase-3 protein were assessed by western blotting; $\beta$-actin was used as the internal control. Data are presented as the mean $\pm \mathrm{SD}(\mathrm{n}=3)$. Statistical significance was determined by one-way ANOVA followed by Dunnett's test. "P<0.05 vs. the CB20 group. LIUS, low-intensity ultrasound; CBP, carboplatin; MGMT, $O^{6}$-methylguanine DNA methyltransferase.

in the mouse model, and determined that Gadd $45 \alpha$ was upregulated in hamster tongue cancer tissues compared with normal tissues (data not shown). Following treatment with CBP, the expression of Gadd $45 \alpha$ in the cytoplasm and nucleus of the control group increased, with a higher increase observed in the nucleus. IHC semi-quantitative and western blot analysis of Gadd $45 \alpha$ protein levels revealed higher Gadd $45 \alpha$ expression in the U+CB group compared to the CB20 group alone (Fig. 4B).

Effect of LIUS combined with CBP on the expression of Chkl, cyclin D1 and cyclin B1. CBP is a cell cycle non-specific drug that can kill cells in different phases of the cell cycle. As shown in Fig. 4 LIUS enhanced CBP damage to the tumor cell DNA. Chk1 is a cell cycle checkpoint kinase that functions in the repair of damaged DNA by blocking the cell cycle after DNA damage. To determine its effects on the cell cycle checkpoints, we examined the expression of Chk1. In the control group, Chk1 was highly expressed in the cytoplasm of the tumor cells, with only a small amount of expression in the nucleus (Fig. 4A). The overall expression of Chk1 in the treatment group was lower than that in the control group; its expression decreased in the cytoplasm and increased in the nucleus with the increase in drug concentration. There were significant differences in Chk1 expression between the CB20 and $\mathrm{U}+\mathrm{CB}$ groups (Fig. 4B; $\mathrm{P}<0.05$ ), indicating that LIUS enhanced the inhibitory effects of CBP on Chk1. Cyclin D1 and cyclin B1 expression was present at lower levels in the $\mathrm{U}+\mathrm{CB}$ group than in the other groups (Fig. 4; $\mathrm{P}<0.05$ ). 

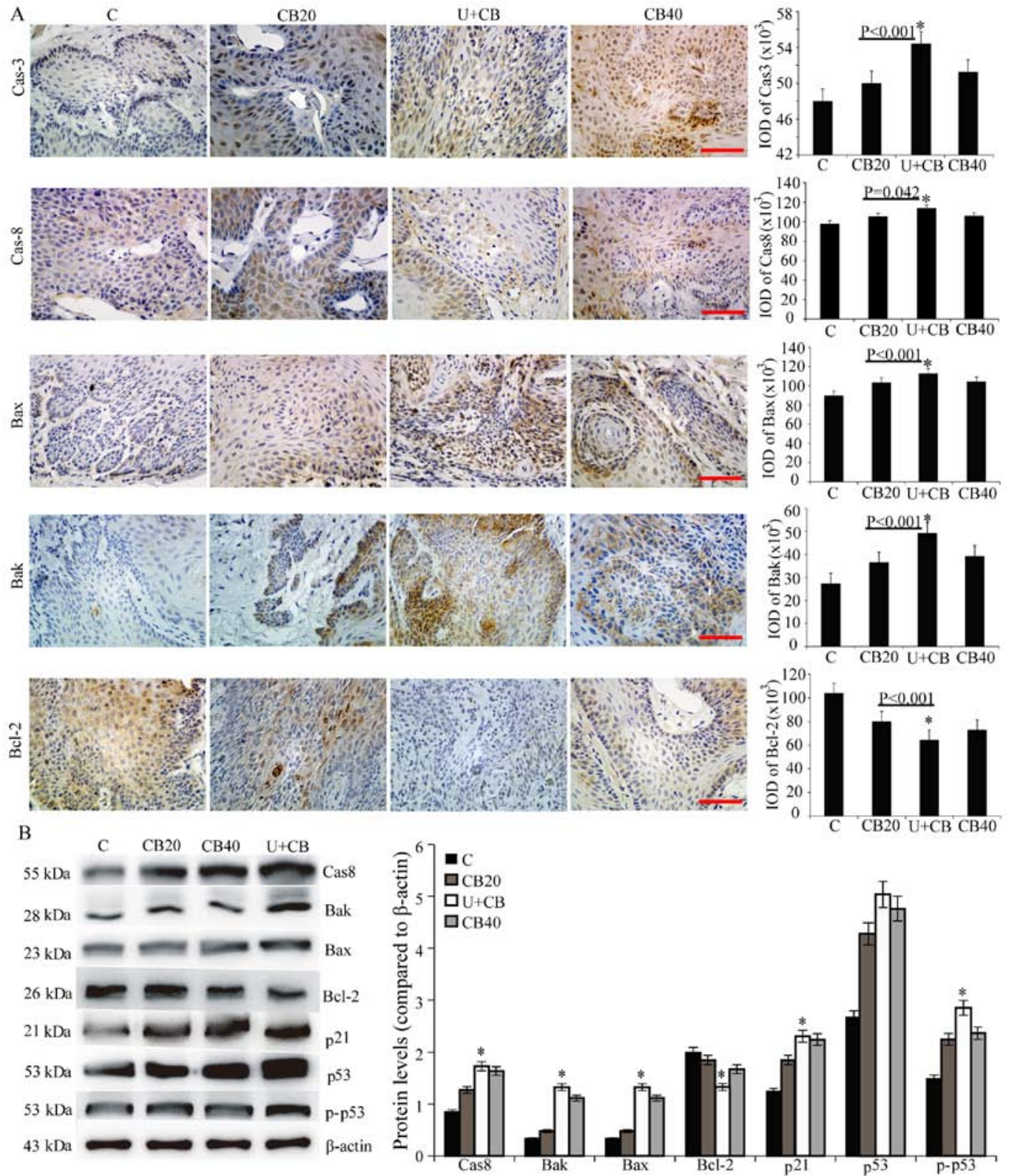

Figure 5. Effect of $\mathrm{U}+\mathrm{CB}$ on the expression of apoptosis proteins in the hamster orthotopic tongue cancer model. (A) Caspase-3, caspase-8, Bak, Bax, and Bcl-2 expression was evaluated by immunohistochemical staining. Representative data from three independent experiments are shown. Scale bar, $50 \mu \mathrm{m}$. (B) The expression levels of caspase-8, Bak, Bax, Bcl-2, p21, p53 and phospho-p53 protein were assessed by western blotting; $\beta$-actin was used as the internal control. Data are presented as the mean $\pm \mathrm{SD}(\mathrm{n}=3)$. Statistical significance was determined by one-way ANOVA followed by Dunnett's test. "P<0.05 vs. CB20 group. $\mathrm{CPB}$, carboplatin; C, control group; U, low-level ultrasound treatment group; CB20, $20 \mathrm{mg} / \mathrm{kg}$ CBP treatment group; CB40, $40 \mathrm{mg} / \mathrm{kg}$ CBP treatment group; $\mathrm{U}+\mathrm{CB}$, low-level ultrasound combined with the $20 \mathrm{mg} / \mathrm{kg}$ CBP treatment group.

Effect of LIUS combined with CBP treatment on the apoptosis of tongue cancer cells. We revealed that LIUS combined with CBP could enhance the DNA damage produced by CBP in tumor cells, and inhibit the expression of Chk1. Then, we determined if these effects eventually led to apoptosis. The expression levels of caspase-3, cleaved caspase-3, caspase-8, $\mathrm{Bax}$, and Bak in the U+CB group were significantly higher than those in the CBP groups, although the expression of $\mathrm{Bcl}-2$ was inhibited (Figs. 4 and 5). We examined the expression levels of wild-type p53 and phospho-p53 in the tissues and determined that although there was no significant difference in total $\mathrm{p} 53$ protein levels among the treated groups, treatment with U+CB significantly increased the level of phospho-p53 compared with the $20 \mathrm{mg} / \mathrm{kg}$ CBP group (Fig. 5B). In addition, there was a significant difference in the expression level of $\mathrm{p} 21$ between the U+CB and other treatment groups (Fig. 5B).

Expression of MGMT, Gadd45a, and Chkl in non-cancerous and OTSCC tissues. In order to anticipate the potential of chemotherapy combination with LIUS in OTSCC patients, it was important to detect whether some tumor markers were overexpressed in human OTSCC samples. We evaluated the expression of MGMT, Gadd $45 \alpha$, and Chk1 in 48 pairs of OTSCC tissue and adjacent non-cancerous oral tissue samples. The representative immunostaining profiles of MGMT, Gadd $45 \alpha$, and Chk1 in OTSCC are displayed in Fig. 6. The immunoreactivities of MGMT, Gadd45 $\alpha$, and Chk1 were increased in OTSCC compared with matched adjacent non-cancerous oral 


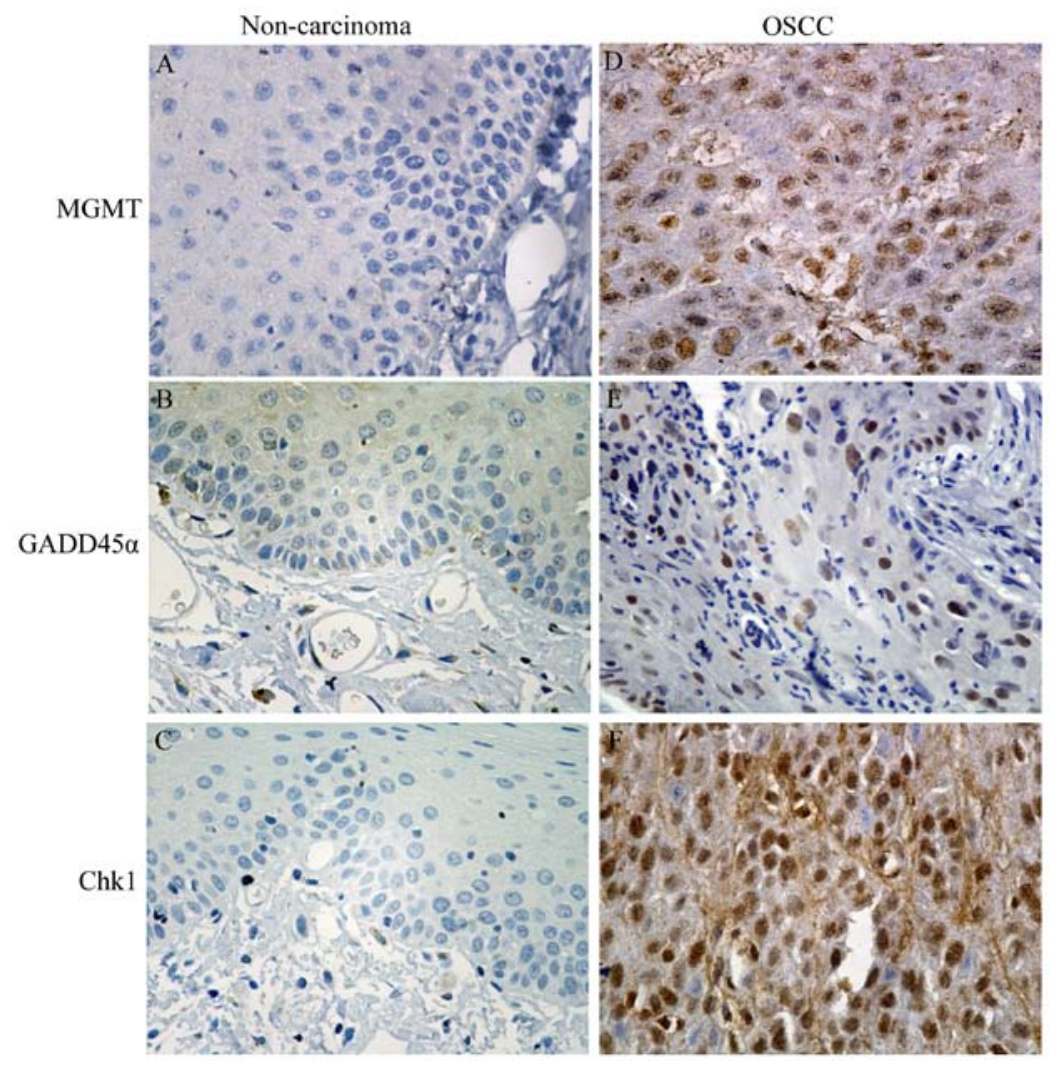

Figure 6. The expression of MGMT, GADD45 $\alpha$, and Chk1 in (A-C) non-carcinoma tissues and (D-F) OTSCC tissues (x400, magnification). MGMT, $O^{6}$-methylguanine DNA methyltransferase; OTSCC, oral tongue squamous cell carcinoma.

tissues. Gadd45 $\alpha$ was widely expressed in OTSCC and adjacent epithelium and was mainly expressed in the nucleus of the adjacent epithelium with a positive expression rate of $100 \%$, whereas there was almost no expression of MGMT and Chk1 in the normal epithelium, and their positive expression rates in OTSCC tissues were 62.5 and $77.1 \%$, respectively. These results revealed that the expression of MGMT, Gadd45a, and Chk1 were upregulated in OTSCC tissues compared with noncancerous tissues, which may provide a convincing rationale for considering LIUS combination with CBP treatment in future clinical studies since our hamster study revealed that the $\mathrm{U}+\mathrm{CB}$ treatment can effectively reduce their expression.

\section{Discussion}

LIUS can enhance the sensitivity of drug-resistant cells to drugs, thereby providing a therapeutic opportunity for multidrug resistance in tumors $(6,10,28)$. Studies have revealed that the use of LIUS combined with low doses of anticancer drugs can achieve the same anticancer therapeutic effects as higher drug doses $(3,4,11)$, however, published studies only used cultured cell levels or nude mice transplanted with tumors. In the present study, we used DMBA to induce an orthotopic hamster model of tongue cancer, and for the first time, used this model to study the effects of LIUS combined with a marginal dosage of $20 \mathrm{mg} / \mathrm{kg}$ CBP on tongue cancer. Our data revealed that LIUS enhanced the in vivo chemotherapeutic effects of CBP without increasing its toxicity. It also prolonged the survival time of the hamsters, indicating that this therapy was safe in the hamster orthotopic model of tongue cancer
(Figs. 2 and 3; Table I). In the present study, we used very low acoustic energy combined with a low dose of CBP to treat an orthotopic hamster model of tongue carcinoma, which is similar to human tongue cancer, allowing for easy translation from pre-clinical to clinical studies.

GADD $45 \alpha$ was identified as a tumor suppressor of multiple types of solid tumors, and patients with high expression of Gadd45 $\alpha$ had better prognosis (29). Our results revealed that the expression of Gadd45 $\alpha$ was higher in cancer tissues compared with adjacent non-cancerous tissues (Fig. 5), which was consistent with a study from Zhang et al (29). Drug therapy can directly or indirectly upregulate GADD $45 \alpha$, promote apoptosis, and increase drug sensitivity (30). We found that the expression of Gadd $45 \alpha$ was increased in hamster tongue cancer after the $\mathrm{U}+\mathrm{CB}$ treatment (Fig. 4), suggesting that LIUS combined with CBP may induce Gadd $45 \alpha$ expression to inhibit cell growth and induce apoptosis (28).

MGMT is a DNA-repair protein, and its expression level is closely related with the sensitivity of cells to drugs $(31,32)$. Chen et al observed that MGMT protected nasopharyngeal carcinoma cells from CDDP-induced DNA damage by enhancing DNA repair capacity, whereas low expression of MGMT in these cells made them more sensitive to platinumbased drugs (33). We found that U+CB led to a decreased expression of MGMT compared to treatment using CBP alone, suggesting decreased DNA repair activity. In terms of the mechanism of LIUS function, further studies are needed in the future to determine if it promotes drug entry into cells, thereby increasing intracellular drug levels $(4,6,11)$ or reduces the threshold of cell destruction (34). 
Whether the cell is apoptotic after cell DNA damage is a critical factor in determining if the damaged DNA can be repaired (35). The ATM/ATR-Chk1-p53-p21/Gadd45 $\alpha$ pathway can regulate cell cycle conversion and the repair of DNA damage (28). As a cell cycle checkpoint kinase, Chk1 undergoes dynamic nuclear-cytoplasmic shuttling under conditions of both normal growth and DNA damage (36). We observed that Chk1 distribution underwent 'cytoplasmicnuclear' shuttling in the U+CB group in that its expression decreased in the cytoplasm (Fig. 4), indicating that there was reduced ability to regulate the DNA damage checkpoint pathway, leading to reduced DNA repair (36) and increased sensitivity to DNA damaging agents (37). The damaged DNA that cannot be repaired leads to the accumulation of genome mutations in cancer cells, resulting in higher sensitivity of cancer cells to chemotherapy than normal cells. Thus, weakening the DNA damage repair ability can improve the sensitivity to chemotherapy, leading to better treatment outcomes (38). The tumor suppressor p53 can inhibit the cancer cell cycle, thereby affecting DNA damage-induced apoptosis (39). We observed that the expression of phosphop53 and p21 increased in orthotopic tongue cancer tissue, and the expression of cyclin D1 and cyclin B1 decreased in the U+CB treatment group (Fig. 5). In addition, the expression of caspase-3, cleaved caspase-3, caspase-8, Bax, and Bak increased and the expression of Bcl-2 protein decreased (Fig. 5), suggesting that LIUS plus CBP treatment may activate the downstream genes p21 and Gadd $45 \alpha$ by inducing wildtype p53 expression, enhancing cell cycle arrest and inducing apoptosis $(40,41)$. As a cell-cycle non-specific drug, CBP can lead to cell cycle arrest, and trigger apoptosis. We determined that the expression levels of cyclin B1 and cyclin D1 were decreased after CBP treatment. We did not perform further in-depth study on the role of CBP on the cell cycle since the focus of this paper was mainly on DNA damage. We will focus on this issue in follow-up experiments.

The most prominent mode of CBP action is the induction of the intrinsic apoptotic pathway through the activation of the DNA damage response (16). The results of our study revealed that LIUS combined with CBP treatment enhanced the cytotoxicity of $\mathrm{CBP}$ in orthotopic tongue cancer by enhancing CBP-mediated DNA damage in tongue cancer cells and inducing apoptosis. To evaluate the clinical relevance of LIUS combined with chemotherapy for treatment of the orthotopic tongue cancer animal model, we collected samples of clinical OTSCC tissue for MGMT, Gadd45 $\alpha$, and Chk1 staining. We found that the expression of MGMT and Chk1 was significantly higher in the OTSCC tissue than in the adjacent tissue, and the expression of Gadd $45 \alpha$ was very low in the cytoplasm of the tumor cells (Fig. 6). Wang et al (42) reported that low MGMT expression resulted in a better prognosis in glioblastoma patients. Gadd $45 \alpha$ significantly increased the chemosensitivity of anticancer drugs (etoposide, cisplatin, and 5 -fluorouracil) in pancreatic cancer cells due to the induction of abundant apoptosis and cell cycle arrest (30). The prognosis of OSCC patients with high Gadd $45 \alpha$ expression was better than those with low expression (29). ATR-Chk1-p53 signal activation was involved in the efficacy of CDDP in OSCC cells (43). These studies support our findings, suggesting that the regulation of cell damage-related factors can enhance the
anti-OSCC ability of CBP and inhibit the malignant progression of OSCC.

Due to hamsters having different sensitivities to DMBA, leading to differences in tumor development time, tumor shape, and tumor growth pattern, difficulties in calculating the tumor size can arise. The LIUS treatment takes time, needs very skilled people to perform, and can only be performed on a limited number of animals according to ethical regulations. The number of surviving animals in the control and LIUS-treated groups are few at the end of the therapy cycle, which limits our ability to perform more analysis. In future experiments, we will increase the number of animals in subsequent experiments to obtain more samples to detect their molecular changes. Despite various limitations in our experiments, the achieved significant inhibition of primary tumor growth was truly encouraging. Compared with CBP treatment alone, $\mathrm{U}+\mathrm{CB}$ treatment did not increase side-effects, demonstrating that this combination therapy is safe, effective and feasible. Thus combining ultrasound with CBP can be a very promising modality for clinical treatment of OSCC. In future studies, we plan to refine the evaluation of tumor growth and increase the number of animals for survival observations.

In conclusion, LIUS enhanced the ability of low-dose CBP to damage DNA in orthotopic tongue cancer cells, induced apoptosis, inhibited tumor growth and progression, and concomitantly did not increase drug side-effects to normal tissues. Thus, combining ultrasound with chemotherapy can be a very promising modality for clinical treatment of tumors.

\section{Acknowledgements}

We would like to thank Editage for providing editorial assistance. This study was supported by grants from the National Natural Science Foundation of China (no. 81502644), the Fundamental Research Funds for the Provincial Universities (no. 2017JCZX28).

\section{Competing interests}

The authors declare that they have no competing interests.

\section{References}

1. Gharat SA, Momin M and Bhavsar C: Oral squamous cell carcinoma: Current treatment strategies and nanotechnology-based approaches for prevention and therapy. Crit Rev Ther Drug Carrier Syst 33: 363-400, 2016.

2. Scully $\mathrm{C}$ and Bagan J: Oral squamous cell carcinoma overview. Oral Oncol 45: 301-308, 2009.

3. Li H, Fan H, Wang Z, Zheng $\mathrm{J}$ and Cao W: Potentiation of scutellarin on human tongue carcinoma xenograft by low-intensity ultrasound. PLoS One 8: e59473, 2013.

4. Hu Z, Lv G, Li Y, Li E, Li H, Zhou Q, Yang B and Cao W: Enhancement of anti-tumor effects of 5-fluorouracil on hepatocellular carcinoma by low-intensity ultrasound. J Exp Clin Cancer Res 35: 71, 2016.

5. Zhou S, Yu T, Zhang J, Liu S, Huo Y and Zhang Y: Sonochemotherapy inhibits the adhesion, migration and invasion of human ovarian cancer cells with highly metastatic potential. Ultraschall Med 32 (Suppl 1): S14-S20, 2011.

6. Yu T, Li SL, Zhao JZ and Mason TJ: Ultrasound: A chemotherapy sensitizer. Technol Cancer Res Treat 5: 51-60, 2006.

7. Trendowski M, Yu G, Wong V, Acquafondata C, Christen T and Fondy TP: The real deal: Using cytochalasin B in sonodynamic therapy to preferentially damage leukemia cells. Anticancer Res 34: 2195-2202, 2014.

8. Trendowski M: The promise of sonodynamic therapy. Cancer Metastasis Rev 33: 143-160, 2014. 
9. Yu T, Yang Y, Zhang J, He H and Ren X: Circumvention of cisplatin resistance in ovarian cancer by combination of cyclosporin A and low-intensity ultrasound. Eur J Pharm Biopharm 91: 103-110, 2015.

10. Zhang Z, Xu K, Bi Y, Yu G, Wang S, Qi X and Zhong H: Low intensity ultrasound promotes the sensitivity of rat brain glioma to Doxorubicin by down-regulating the expressions of p-glucoprotein and multidrug resistance protein 1 in vitro and in vivo. PLoS One 8: e70685, 2013.

11. Kotopoulis S, Delalande A, Popa M, Mamaeva V, Dimcevski G, Gilja OH, Postema M, Gjertsen BT and McCormack E: Sonoporation-enhanced chemotherapy significantly reduces primary tumour burden in an orthotopic pancreatic cancer xenograft. Mol Imaging Biol 16: 53-62, 2014.

12. Yu FT, Chen X, Wang J, Qin B and Villanueva FS: Low intensity ultrasound mediated liposomal doxorubicin delivery using polymer microbubbles. Mol Pharm 13: 55-64, 2016.

13. Rizzitelli S, Giustetto P, Faletto D, Delli Castelli D, Aime S and Terreno E: The release of Doxorubicin from liposomes monitored by MRI and triggered by a combination of US stimuli led to a complete tumor regression in a breast cancer mouse model. J Control Release 230: 57-63, 2016.

14. Lorusso D, Petrelli F, Coinu A, Raspagliesi F and Barni S: A systematic review comparing cisplatin and carboplatin plus paclitaxel-based chemotherapy for recurrent or metastatic cervical cancer. Gynecol Oncol 133: 117-123, 2014.

15. Amable L: Cisplatin resistance and opportunities for precision medicine. Pharmacol Res 106: 27-36, 2016.

16. Dasari S and Tchounwou PB: Cisplatin in cancer therapy: Molecular mechanisms of action. Eur J Pharmacol 740: 364-378, 2014.

17. Stewart DJ: Mechanisms of resistance to cisplatin and carboplatin. Crit Rev Oncol Hematol 63: 12-31, 2007.

18. Saad AH and Hahn GM: Ultrasound enhanced drug toxicity of Chinese hamster ovary cells in vitro. Cancer Res 49: 5931-5934, 1989.

19. Sausville EA and Burger AM: Contributions of human tumor xenografts to anticancer drug development. Cancer Res 66: 3351$3354,2006$.

20. Bibby MC: Orthotopic models of cancer for preclinical drug evaluation: Advantages and disadvantages. Eur J Cancer 40: 852-857, 2004.

21. Teicher BA: Tumor models for efficacy determination. Mol Cancer Ther 5: 2435-2443, 2006.

22. Fan H, Jiang W, Li H, Fang M, Xu Y and Zheng J: MMP-1/2 and TIMP-1/2 expression levels, and the levels of collagenous and elastic fibers correlate with disease progression in a hamster model of tongue cancer. Oncol Lett 11: 63-68, 2016.

23. Zheng J, Xie L, Teng H, Liu S, Yoshimura K, Kageyama I and Kobayashi K: Morphological changes in the lingual papillae and their connective tissue cores on the 7,12-dimethylbenz[alpha] anthracene (DMBA) stimulated rat experimental model. Okajimas Folia Anat Jpn 85: 129-137, 2009.

24. Fujita K, Kaku T, Sasaki M and Onoé T: Experimental production of lingual carcinomas in hamsters: Tumor characteristics and site of formation. J Dent Res 52: 1176-1185, 1973.

25. Zheng J, Wang Q, Li H, et al: Comparative study on the tongue carcinoma model induced by local application both of 7,12-dimethylbenz[a]anthracene (DMBA) and injury. Anatomy Res 29: 405-409, 2007.

26. Lv Y, Zheng J, Zhou Q, Jia L, Wang C, Liu N, Zhao H, Ji H, Li B and Cao W: Antiproliferative and apoptosis-inducing effect of exo-protoporphyrin IX based sonodynamic therapy on human oral squamous cell carcinoma. Sci Rep 7: 40967, 2017.

27. Fan HX, Li HX, Chen D, Gao ZX and Zheng JH: Changes in the expression of MMP2, MMP9, and ColIV in stromal cells in oral squamous tongue cell carcinoma: Relationships and prognostic implications. J Exp Clin Cancer Res 31: 90, 2012.

28. Zhang Z, Chen J, Chen L, Yang X, Zhong H, Qi X, Bi Y and $\mathrm{Xu} \mathrm{K}$ : Low frequency and intensity ultrasound induces apoptosis of brain glioma in rats mediated by caspase- $3, \mathrm{Bcl}-2$, and survivin. Brain Res 1473: 25-34, 2012.
29. Zhang XY, Xun-Qu, Wang CQ, Liu GX, Zhou CJ and Wang ZG: Expression of growth arrest and DNA damage inducible 45a in human oral squamous cell carcinoma is associated with tumor progression and clinical outcome. J Cancer Res Ther 10 (Suppl): C108-C113, 2014.

30. Li Y, Qian H, Li X, Wang H, Yu J, Liu Y, Zhang X, Liang X, Fu M, Zhan Q and Lin C: Adenoviral-mediated gene transfer of Gadd45a results in suppression by inducing apoptosis and cell cycle arrest in pancreatic cancer cell. J Gene Med 11: 3-13, 2009.

31. Pegg AE: Multifaceted roles of alkyltransferase and related proteins in DNA repair, DNA damage, resistance to chemotherapy, and research tools. Chem Res Toxicol 24: 618-639, 2011.

32. Christmann M, Verbeek B, Roos WP and Kaina B: O(6)Methylguanine-DNA methyltransferase (MGMT) in normal tissues and tumors: Enzyme activity, promoter methylation and immunohistochemistry. Biochim Biophys Acta 1816: 179-190, 2011.

33. Chen SH, Kuo CC, Li CF, Cheung CH, Tsou TC, Chiang HC, Yang YN, Chang SL, Lin LC, Pan HY, et al: O6-methylguanine DNA methyltransferase repairs platinum-DNA adducts following cisplatin treatment and predicts prognoses of nasopharyngeal carcinoma. Int J Cancer 137: 1291-1305, 2015.

34. Yu T, Xiong Z, Chen S and Tu G: The use of models in 'target theory to evaluate the survival curves of human ovarian carcinoma cell line exposure to adriamycin combined with ultrasound. Ultrason Sonochem 12: 345-348, 2005.

35. Wang L, Gao S, Jiang W, Luo C, Xu M, Bohlin L, Rosendahl M and Huang W: Antioxidative dietary compounds modulate gene expression associated with apoptosis, DNA repair, inhibition of cell proliferation and migration. Int J Mol Sci 15: 16226-16245, 2014.

36. Wang J, Han X, Feng X, Wang Z and Zhang Y: Coupling cellular localization and function of checkpoint kinase 1 (Chk1) in checkpoints and cell viability. J Biol Chem 287: 25501-25509, 2012.

37. Zhang $\mathrm{Y}$ and Hunter T: Roles of Chk1 in cell biology and cancer therapy. Int J Cancer 134: 1013-1023, 2014.

38. Jackson SP and Helleday T: DNA REPAIR. Drugging DNA repair. Science 352: 1178-1179, 2016.

39. Wang X, Simpson ER and Brown KA: p53: Protection against tumor grow th beyond effects on cell cycle and apoptosis. Cancer Res 75: 5001-5007, 2015.

40. Cheng SY, Seo J, Huang BT, Napolitano T and Champeil E: Mitomycin $\mathrm{C}$ and decarbamoyl mitomycin $\mathrm{C}$ induce p53-independent p21 $1^{\text {WAF1/CIP1 }}$ activation. Int $\mathrm{J}$ Oncol 49: 1815-1824, 2016

41. Canne IG, Merrick KA, Morandell S, Zhu CQ, Braun CJ, Grant RA, Cameron ER, Tsao MS, Hemann MT and Yaffe MB: A pleiotropic RNA-binding protein controls distinct cell cycle checkpoints to drive resistance of $p 53$-defective tumors to chemotherapy. Cancer Cell 28: 831, 2015.

42. Wang W, Zhang L, Wang Z, Yang F, Wang H, Liang T, Wu F, Lan Q, Wang J and Zhao J: A three-gene signature for prognosis in patients with MGMT promoter-methylated glioblastoma. Oncotarget 7: 69991-69999, 2016.

43. Hung CC, Chien CY, Chiang WF, Lin CS, Hour TC, Chen HR, Wang LF, Ko JY, Chang CH and Chen JY: p22phox confers resistance to cisplatin, by blocking its entry into the nucleus. Oncotarget 6: 4110-4125, 2015.

This work is licensed under a Creative Commons Attribution-NonCommercial-NoDerivatives 4.0 International (CC BY-NC-ND 4.0) License. 